Steroid was used by $39.2 \%$ of patients with diabetes and hypertension. Cardiovascular events (Ischemic heart disease and ischemic stroke) occurred in $2.2 \%$ of patients and $45.5 \%$ of patients with cardiovascular events were receiving concomitant steroid. Infections requiring hospital visit were recorded in $1.8 \%$ of patients; $77.8 \%$ of patients with infection were on biologic DMARD and $33.3 \%$ were receiving concomitant steroid.

Out of Two hundred seventy six patients who underwent DXA scanning for estimation of bone mineral density, $48.6 \%$ were having decrease bone density ( $37 \%$ osteopenia, $11.6 \%$ osteoporosis).

Steroid use was significantly associated with decrease bone density.

Conclusions: Comorbid conditions are frequently associated with Rheumatoid arthritis as observed in our cohort of patients. Patient care should not be focused only on arthritis care. All RA patients should be screened for comorbidities and treated accordingly in order to avoid their deleterious effect on patient health.

Disclosure of Interest: None declared

DOI: 10.1136/annrheumdis-2017-eular.1899

\section{FRI0759-HPR COLLABORATION BETWEEN GENERAL PRACTITIONERS AND RHEUMATOLOGISTS TO MANAGE CARDIOVASCULAR RISK IN PATIENTS WITH RHEUMATOID ARTHRITIS PATIENTS}

J. Weijers $^{1}$, S. Rongen-van Dartel ${ }^{1,2}$, M. Hulscher ${ }^{1}$, P. van Riel ${ }^{1,2}{ }^{1} / Q$ healthcare, Scientific Institute for Quality of Healthcare, Radboud university medical center, Nijmegen; ${ }^{2}$ Department of Rheumatology, Bernhoven, Uden, Netherlands

Background: To reduce the risk of cardiovascular disease in rheumatoid arthritis (RA) patients, adequate cardiovascular risk management (CVRM) is necessary. CVRM implies assessment, treatment and monitoring of cardiovascular risk factors ${ }^{1}$. The updated EULAR guideline states that cardiovascular risk assessment should be considered at least once every five years in all patients with $\mathrm{RA}^{2}$. A few studies show suboptimal risk management in daily practice in selected groups of patients.

Objectives: This study aims to describe current performance of the CVRM recommendation in a hospital based RA population in the South of the Netherlands. In this region, general practitioners (GPS) and rheumatologists closely collaborate into manage RA patients' cardiovascular risk.

Methods: Due to the collaboration, CVRM is performed as a part of a transmural care program. The rheumatologist informs the GP when a patient has been diagnosed with RA. The patient is placed on a list for CVRM to be screened by a specialised nurse practitioner. As a part of the collaboration, laboratory results requested by the GPS and rheumatologists are collected in one digital patient record system. This system is used to check whether the RA patient's lipid profile was determined in the previous five years. If not, a letter with the listed patient is sent to the GP a reminder for screening the patient. In this study, we checked six months later whether lipid testing was ultimately performed.

Results: In 70\% ( $n=475$ ) of all 679 RA patients (mean age 63 (SD 9 years), 68\% women and median disease duration of 7 years (IQR 3-11)) a lipid profile was determined in the previous five years.

Of the 204 non-screened RA patients, 98 had been screened after sending the letter to their GP (+48\%), see Figure 1. No differences in gender and disease duration were found between the screened and non-screened patients $(p=.46$ and $p=.25$ respectively). By contrast screened patients were 10 years older compared to the non-screened patients (66 year (SD12) vs 56 (SD 15) year, $p<0.0001$ ).

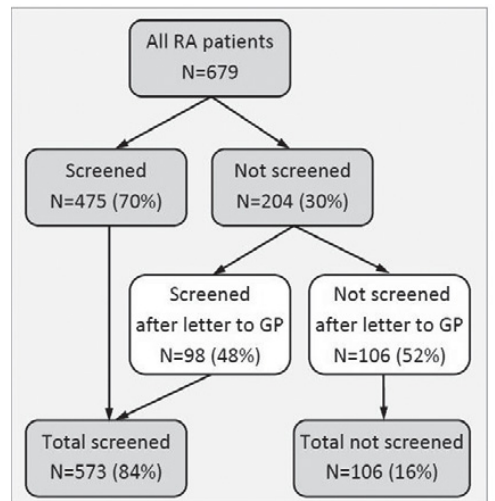

Figure 1 Flowchart screening all rheumatoid arthritis (RA) patients for their cardiovascular risk; results of sending a letter to general practitioners (GP).

Conclusions: As a result of the collaboration between GPs and rheumatologists, $70 \%$ of all RA patients was screened for CVRM. A small intervention, sending a reminding letter to the GP, increased this percentage even further, to $84 \%$. This collaboration can be seen as a good practice to provide care in line with the EULAR guideline.

References:

[1] Barber CE et al. Best practices for cardiovascular disease prevention in rheumatoid arthritis: a systematic review of guideline recommendations and quality indicators. Arthritis care \& research. 2015;67(2):169-79.

[2] Agca $R$ et al. EULAR recommendations for cardiovascular disease risk management in patients with rheumatoid arthritis and other forms of inflammatory joint disorders: 2015/2016 update. Annals of the rheumatic diseases. 2016

Disclosure of Interest: None declared

DOI: 10.1136/annrheumdis-2017-eular.2423

\section{FRI0760-HPR BIOSIMILAR USE AMONG EUROPEAN RHEUMATOID ARTHRITIS PATIENTS AND IMPACT ON PATIENT OUTCOMES}

L. Chanroux, F. Mboge, A. Wadiwalla. Therapy Watch, Research Partnership, London, United Kingdom

Background: Biologic agents have been shown to help control disease progression in rheumatoid arthritis (RA) and significant reduce joint damage. However, their considerable cost has limited their widespread use.

Objectives: Biosimilars offers the opportunity for significant cost savings for national health services and the aim of this research is to better understand their use among European rheumatologists and their potential impact on patient outcomes.

Methods: We used data collected as part of an online treatment survey conducted among a panel of 261 rheumatologists between January and December 2016 across 5 European markets (France, Germany, Italy, Spain and the UK). Physicians were sampled to provide a representative mix of practice types and regions. Our record form sample included 9,650 patient currently treated with a bDMARD, 297 of which received a biosimilar. We split the sample into 2 groups, biosimilar patients $=$ those treated with Benepali (etanercept), Remsima (infliximab), Inflectra (infliximab) and Flixabi (infliximab) and originator patients $=$ those treated with Enbrel (etanercept) or Remicade (infliximab). We analysed patient demographic data along with current DAS, joint count, HAQ score and perceived disease severity to assess response to therapy over time.

Results: Biosimilars accounted for a total of $2.4 \%$ of our biologic sample with the greatest uptake of these agents reported in the UK $(3.8 \%)$ and the lowest in France $(1.6 \%)$. Use of biosimilars increased in patients who started their current biologic in $2016(8.1 \%)$, with a marginally higher use seen in patients on their 2nd or higher line of bDMARD therapy vs. those on their 1 st bDMARD $(9.4 \%$ vs. $7.5 \%$, respectively).

We saw no significant difference in the distribution of biosimilar and originator patients by age and gender although biosimilar patients appeared to have more severe disease. While a smaller proportion of biosimilar patients were perceived to have moderate/severe RA at diagnosis $(79.1 \%$ vs. $88.4 \%)$ a greater proportion were thought to have moderate/severe disease at their latest visit $(64.3 \%$ vs. $44.2 \%)$. The average DAS28 of biosimilar patients was higher at a directional level but their average HAQ score and tender/swollen joint count were non-significantly lower. Biosimilar patients were more likely to suffer from a comorbid condition $(87.2 \%$ vs. $74.1 \%)$ and a autoimmune condition beyond their RA (17.2\% vs. $11.6 \%$ ). There were no significant differences in the proportion of patients unable to work due to their disease $(4.3 \%$ on average from the total sample).

We analysed the data focusing solely on 1st line patients to reduce any bias introduced by previous lines of therapy but observed similar trends. A higher proportion of biosimilar patients were considered to have moderate to severe RA at their latest visit $(67.9 \%$ vs. $43.0 \%)$ and a greater proportion of patients had a DAS28 > 5.1 (23.3\% vs.3.0\%).

Conclusions: Our research suggests that biosimilar uptake remains limited amongst European rheumatologists with a directional trend towards 2 nd line use. Our data did not clearly show any significant differences in the profile of biosimilar patients or their outcomes. Increased governance from healthcare regulators and additional clinical data may be needed to further establish the efficacy and safety of these agents and drive their wider use, ensuring greater cost efficiency and the potential for wider access to biologic therapies for RA patients.

Disclosure of Interest: None declared

DOI: 10.1136/annrheumdis-2017-eular.6598

\section{FRI0761-HPR CHRONIC WIDESPREAD PAIN PREVALENCE IN THE GENERAL POPULATION: A SYSTEMATIC REVIEW \& META-ANALYSIS}

P. Andrews, M. Steultjens, J. Riskowski. Health and Life Sciences, Glasgow Caledonian University, Glasgow, United Kingdom

Background: Chronic widespread pain (CWP) is a worldwide health problem and a significant contributor to disability. Understanding the impact of individualdependent (e.g., gender) and contextual-dependent (e.g., survey method, latitude) factors have on CWP prevalence may provide a foundation population-based strategy for addressing CWP.

Objectives: To determine a general population worldwide estimate of CWP prevalence and to examine the individual and contextual-dependent factors related to CWP prevalence.

Methods: A systematic review was undertaken using seven databases. Along with data extracted from the manuscripts, additional contextual data including WHO development status and region, human development index (HDI; measure 